\title{
Special issue on health care simulation
}

\section{Preface}

\author{
James G. Anderson • Godefridus G. van Merode
}

Published online: 25 September 2007

(C) Springer Science + Business Media, LLC 2007

Health-related issues continue to be global challenges. Both developed and developing nations are struggling with ways to provide affordable access to health services. The threat of global epidemics including HIV, SARS and the spread of other diseases worldwide threaten to undo decades of gains in life expectancy. Poverty and political unrest breeds new diseases that are rapidly spread around the globe. At the same time the organization and delivery of health services are increasingly complex and costly. Papers in this issue demonstrate the power of simulation in addressing these challenges. Computer simulation has become state of the art in many areas and its use is still growing. Two trends can be observed. First, the conceptual scope of both simulation software and models is becoming broader and more flexible. This allows investigators to combine several modelling approaches in the same application. For example, agentbased models allow researchers to combine technical as well as behavioural aspects of systems. Second, partly as a result of the first trend, we see the use of computer simulation increasingly being applied in areas where there used to be reluctance to use this approach, e.g., in policy modelling.

The first three papers address health care policy issues. Pasdirtz examines the issue of control of health care expenditures in the US. State-space models of the US health care system and the US economy were developed,

J. G. Anderson $(\bowtie)$

Purdue University,

West Lafayette, IN, USA

e-mail: andersonj@purdue.edu

G. G. van Merode

Maastricht University,

Maastricht, The Netherlands estimated with time series data for the late twentieth century and analyzed using modern control theory. The results suggest that control strategies designed to contain health care costs need to be targeted at health care capital investment, prices for physicians' services and prescription drugs. Policies directed purely at cutting costs in general or increasing efficiency are likely to have little effect.

The paper by Anderson and others reports the construction of an agent-based model that has been used to study humanitarian assistance policies executed by governments and non-government organizations (NGOs) for the health and safety of refugees. An experimental design was used to study the relationships among five factors: basic needs, food and water, sanitation, medical resources and security. The simulation demonstrates the critical role of security in providing for the health and well-being of refugees. The importance of security was highlighted in the recommendations that Amnesty International made regarding the African Union Mission in Sudan (AMIS). A major strength of the model is that it allows policy makers to incorporate specific characteristics of refugees and the governmental and nongovernmental organizations that are providing humanitarian aid to the camp. Policy simulations are valuable because of their ability to facilitate training and feedback concerning potential impact of decisions. In the real world policy decisions may have serious consequences. Policy simulation models are useful because they accelerate creation of scenarios, allow rapid changes in parameters, and provide a test bed for concepts and strategies. In the present application, policies can be examined to see how refugee communities might respond to alternative courses of action and how these actions are likely to affect the health and well-being of the community.

The third paper in this issue demonstrates the value of simulation as a tool to analyze policies designed to contain 
epidemics. Armbruster and Brandeau use simulation to examine the effects of different contact tracing policies on the control of infectious disease. Contact tracing is a primary means of controlling infectious diseases such as tuberculosis (TB), HIV, and sexually transmitted diseases (STDs). The authors develop a simulation model of contact tracing and the spread of an infectious disease among members of a network. The model is used to evaluate the cost and effectiveness of different levels of contact tracing. Control of Chlamydia, a prevalent STD, is used to illustrate the model. The authors discuss how simulation could be used as a policy tool for determining the appropriate level of investment in contact tracing for a specific disease in a specific population.

The next three papers apply simulation to a range of health care challenges that arise in the design of health care systems. Creating optimal processes or system designs is a challenge because health care involves a tradeoff between the level of process efficiency, costs, patient values and employee values. These papers demonstrate the role that simulation plays in design decisions including the design of hospitals to support efficient delivery of health care services and part-time work by physicians and the reduction of wait times for general surgery.

Vos and colleagues describe a methodology that can be used to evaluate hospital design to assure that it supports efficient and effective health care processes. A discrete event simulation model is developed representing flow of people and goods through the hospital facility. An experimental design is applied to assess the effects of arrival patterns, patient mix and the duration of appointments. This study demonstrates how simulation can be used to bridge architecture and operational control by assessing a hospital design for functionality and its ability to meet future developments.

The paper by Molema and others examines how health care systems can be designed to enable physicians to work part-time. Discrete event simulation was used to model the work process of surgeons providing care for elective patients with intestinal problems. The model was used to evaluate two strategies for enabling part-time work by physicians. The results suggest that work redesign can enable part-time work and improve system performance. At the same time, systems characterized by different levels of variability require different part-time arrangements.

The study by VanBerkel and Blake explores ways to maximize throughput in general surgery and reduce wait times with current levels of resources. A discrete event simulation model was constructed to aid capacity planning decisions and to analyze performance. The simulation showed that wait times are more dependent on beds than on available OR time. Wait time can be reduced by redistributing beds between sites and achieving standard patient lengths of stay.

Computer simulation has begun to augment traditional mathematical approaches to modeling that impose serious restrictions due to their assumptions. These assumptions include linearity, homogeneity, normality, and stationarity. The papers included in this issue apply state-space models, discrete event simulation and agent-based modeling. Addressing health-related challenges requires methodologies that aid decision making. The challenge to the simulation community is to develop methods and applications that provide insight and that have an impact on local, national and international policies. 